\title{
Toll-Like Receptor-4, Synthesis is Regulated by JNK Signaling, by Three Glucocorticoids Isoforms and by Three Interferons Isoforms, also its Advantages in Activities Depending on the Containment from Arg, Proline and Hydrophobic Amino Acids in its Compositions
}

\author{
Ashraf Marzouk El Tantawi* \\ Department of Biomedical molecular studies, Egypt
}

\begin{abstract}
Toll-like receptor-4 (TLR4), synthesis is regulated by JNK signaling, by three glucocorticoids isoforms, and by the three interferons isoforms, also depending on availabilities of LPS \& on long fatty acids chains with Arg and proline availabilities. For performing and running the mitochondrial oxidative processes for producing fatty-acyl-CoA-synthetase followed by fatty-acyl-CoAsynthase followed by fatty-acyl-CoA-phospholipase productions for linear $\mathrm{TLR}_{4}$ active beta-subunits which will be transformed into $\mathrm{TLR}_{4}$-alpha upon phospholipase effects, which will follow phosphorylation process (alpha-oxidations) for generate Guanosine triphosphate cyclopyrrolone (GTP-Chase) subunits which supposed to contain specific hydrophobic amino acids including Arg, Tyr, leu, proline.... etc., which is the rate-limiting enzyme for tetrahydrobiopterin $\left(\mathrm{BH}_{4}\right)$ synthesis, which is essential for inducible iNOS from fatty-acyl-CoA-synthase upon the nitric oxide-synthase (NO-S) regulations effects.
\end{abstract}

Proline can accelerate anabolic oxidative processes by $\mathrm{OPA}_{1}$ enzymes and provides site-specific flexibility for collagen synthesis in vivo, and. Plays a necessary important role in $\mathrm{TLR}_{4}$ and TGF-gamma/beta/\& alpha synthesis and activities, where the presence of proline in IFN-gamma, in TLR 4 genes and in IFN-beta will accelerate oxidative OPA anabolic processes and direct the flow of biological processes to proliferations of plasma-membranes, collagen synthesis and blood platelets biosynthesis. Vitamin $\mathrm{E}$ \& K-dependent protein $\mathrm{C}$ are the key components of anticoagulant serine protease, And therefore vit $\mathrm{E}$ and vit $\mathrm{k}$ are providing specific advantages to $\mathrm{TLR}_{4}$ synthesis and modulated first in vivo as proper fatty-acyl-CoA-synthetase subunits (gamma-subunits) then modified fatty-acyl-CoA-synthase subunits upon synthase effects on gamma-subunits for producing IL-beta upon which will promote linear $\mathrm{TLR}_{4}$ upon both synthase and phospholipase effects for starting proliferation steppes started by catalyzing Arg for producing GTP-Chase and citrulline which the main basis for Erythropoietin productions, for Plasma membrane synthesis... etc.

Both IFN-beta and glucocorticoid-beta are designed anti-inflammatory subunits are depending on each other's and on the activities of $\mathrm{OPA}_{1}$-synthase enzyme for producing the long fatty acyl-CoA-synthase (Beta-subunit) with specific compositions and sequences from amino acids which can determine their advantages in immunity functions eg. their containment of try, proline, Arg, gly. etc., where, both glucocorticoid-beta and IFN-beta can recover each other's in their different tissues.

Quick Response Code:

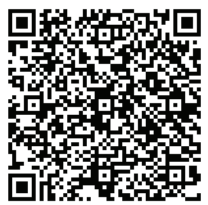

Address for correspondence: Ashraf Marzouk EI Tantawi, Department of Biomedical molecular studies, Egypt

Received: August 19, $2021 \quad$ Published: August 31, 2021

How to cite this article: Ashraf Marzouk El T. Toll-Like Receptor-4, Synthesis is Regulated by JNK Signaling, by Three Glucocorticoids Isoforms and by Three Interferons Isoforms, also its Advantages in Activities Depending on the Containment from Arg, Proline and Hydrophobic Amino Acids in its Compositions. 2021- 3(4) OAJBS.ID.000316. DOI: 10.38125/OAJBS.000316 


\section{MATERIALS}

1) Interferons (IFN-gamma, IFN-beta, IFN-alpha)

2) glucocorticoid-gamma, glucocorticoid-beta, Glucocorticoidalpha

3) Toll-like receptor $\left(\mathrm{TLR}_{4}\right)$ genes

4) the three gamma, beta, and alpha-subunits (fatty-acyl-CoAsynthetase, synthase and phospholipase)

5) Guanosine triphosphate cyclohydrolase

6) tetrahydrobiopterin $\left(\mathrm{BH}_{4}\right)$

7) extracellular linear signal-regulated beta-kinases $\left(\mathrm{ERK}_{1}\right.$ and $\left.\mathrm{ERK}_{2}\right)$

8) lipopolysaccharide (LPS), long fatty acids chains

9) AMPK

10) JNK processes

11) FOX genes, and mitochondrial $\mathrm{OPA}_{1}$ inner membrane enzymes

12) Glutaric acid to ketoglutarate then to glutamate for proline synthesis (TCA cycle)

13) Erythropoietin (EPO)

14) phosphatidylinositol 3-kinase $\left(\mathrm{PI}_{3} \mathrm{~K}\right)$

15) $S_{6} K_{1}$ protein and $23 \mathrm{~S}$ ribosomal gene

\section{INTRODUCTION}

Toll-like receptor-4 $\left(\mathrm{TLR}_{4}\right)$ biosynthesis is activated by lipopolysaccharide bindings and long fatty acids chains, where LPS is necessary for producing firstly long active fatty-acyl-CoAsynthetase upon the effects of synthetase by activating gammaoxidations, where, $\mathrm{TLR}_{4}$ biosynthesis can be run through and depending on RORs biosynthesis after FOX genes functions and activities for producing the four kinases molecules forms (where the FOX activities are for producing the four kinds of kinases each carry specific advantages of activities that direct their own functions in biological pathways that later can be identified as mitogens each has its own amino acids in specific qualities and quantities in arranged sequences for advantages of biological processes ).

So, in Briefly, FOX functions are for preparing and producing specific mitogens mainly in four groups PS/T-kinase, PS/TG-kinase, ps/tt-kinase, \& PS/TA -kinase, but second imp steps is functioning some or all the four previous kinases groups for RORs isoforms synthesis which simply are classified into three active long-fattyacyl-CoA molecules isoforms. Which formed in so classified arranged three steps each step depending on its previous one for running next one and all steppes depending on OPA mitochondrial membrane enzymes which presents in specific orders for running their own processes in advantages of highly arranged controlled effects. Started by gamma-oxidation for producing fatty-acyl-CoAsynthetase (gamma-subunits)upon synthetase effects followed by beta-oxidations for fatty-acyl-CoA-synthase subunits synthesis which upon phospholipase effects which will promote the $\mathrm{TLR}_{4}$ biosynthesis which also can be stimulated by TCA cycles through firstly stimulating the purines synthesis which will stimulate the synthetase activities for adopting purines productions (by
TCA cycles) by pyrimidine synthesis (which run by synthetase for hydrophobic acids production), followed finally by anabolic oxidative processes by $\mathrm{OPA}_{1}$ enzymes for active $\mathrm{TLR}_{4}$ synthesis which through transcriptions will re-activate phospho-lipoproteins synthesis for phospholipid membrane biosynthesis and cells proliferations.

$\mathrm{TLR}_{4}$ functions is the basic tools to Promote the plasmamembrane synthesis and increase its own compositions for signaling receptor activities due to its biosynthesis origin which depend on the four protein kinases compositions and productions started by FOX genes pathways activities (mitogen synthesis ) followed by the three anabolic oxidative processes (gamma, beta, alpha-oxidations) by $\mathrm{OPA}_{1}$ enzymes for first producing gamma-subunits then will be oxidized by synthase for producing Beta-subunits which will be oxidized by phospholipase (alpha oxidations) for $\mathrm{TLR}_{4}$ production, Where, $\mathrm{TLR}_{4}$ is the bases of regulating plasma membrane biosynthesis with specific advantages of compositions, also $\mathrm{TLR}_{4}$ functions is regulating endosome membrane synthesis (which depend on SK6 genes synthesis and on the ribosomes composition) upon the effects of phospholipase on beta-subunits by running for producing active fatty-acyl-CoAphospholipase subunits for phospho-lipo-protein synthesis and for cells proliferation pathways. $\mathrm{TLR}_{4}$ regulate the MHC class II biosynthetic process, and through its feedback that reactivate and adopt the positive regulation of JNK cascade, where JNK signaling processes are the main basis for TLR4 biosynthesis too and for proliferation pathways (Figure 1).

$\mathrm{TLR}_{4}$ also regulate and adopt the interleukins productions and promote the regulation of extracellular linear signal-regulated beta-kinases $\left(\mathrm{ERK}_{1}\right.$ and $\mathrm{ERK}_{2}$ ), For re-activate T-helper 1 cells type immune response, also promote. Platelet biosynthesis pathways through interferon beta and then alpha productions (where Interferons considered as cytokines that through transcriptions will innate immune Response against viral infection and then for types of advantages of cells proliferations), also $\mathrm{TLR}_{4}$ Regulate the $\mathrm{NLRP}_{3}$ inflammasome.

\section{METHODS ARE RESULTS}

Toll-like receptor-4 $\left(\mathrm{TLR}_{4}\right)$ biosynthesis started by the Stimulations of mitogen synthesis through FOX functions processes then followed by the effects of the mitochondria OPA 1 enzymes on availability of long fatty acids chains or on lipopolysaccharide bindings for producing the three types of active subunits isoforms started by gamma subunits upon the effect of synthetase for producing acyl-CoA-synthetase then followed by effect of synthase on gamma-subunits for producing beta-subunits (fatty-acyl-CoAsynthase subunits ) then ended by productions of fatty-acyl-CoAohospholipase (alpha subunits)upon the effects of phospholipase on Beta-subunits, where long fatty acids chains and LPS production in vivo is first upon the effects of ATPase and COX enzymes on lipid, and on lipoproteins which are necessary for activating the productions of firstly the long active fatty-acyl-CoA-synthetase followed by producing the fatty-acyl-CoA-synthase then followed by fatty-acyl-CoA-phospholipase (alpha-subunits) productions upon mitochondrial OPA ${ }_{1}$ enzymes effect, where the alpha-subunits productions is the necessary step for $\mathrm{TLR}_{4}$ synthesis including specific containment from specific amino acids which imp for increasing anti-inflammation, for phospholipid synthesis.

$\mathrm{TLR}_{4}$ can promise the regulations of linear extracellular signal beta-kinases (ERK1/2) that through feedback can re-activate 
gamma-kinases gamma-subunits by gamma-oxidation and further cellular dependent activities which participates in the regulations of many cellular activities including adopting purines results from TCA cycles, and adopting pyrimidines synthesis in vivo by synthetase for hydrophobic acids synthesis, Where both TCA cycles and synthetase activities are cooperating well together for providing the proper purines and pyrimidines for building the proper gamma-subunits (long fatty-acyl-CoA-synthetase) during gamma-oxidation vie synthetase effects, and those two cycles are related to each other and considered necessary for adopting purines and pyrimidines synthesis in vivo.

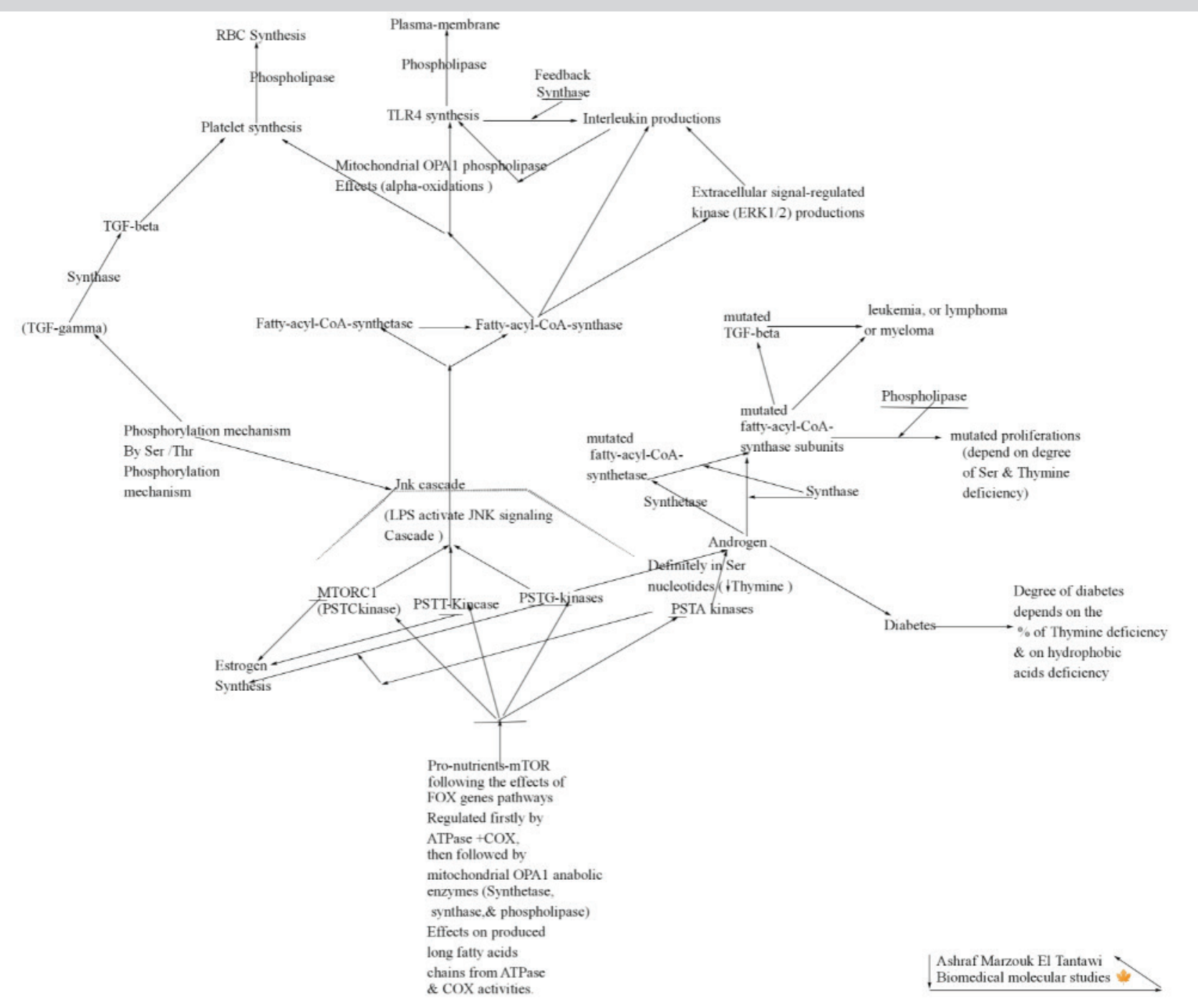

Figure 1: Consider the necessaries of Ser/Thr availabilities in the kinases Proteins and consider the TLR4 synthesis and TGF-beta synthesis.

\section{JNK Signaling Cascade Regulate TLR $_{4}$ Synthesis Depending on Phospholipase Effects}

C-Jun N-terminal kinases (JNKs), identified as subunits proteins kinases were produced firstly by FOX genes activities pathways (where FOX functions are specific for mitogen proteins kinases family synthesis). JNK processes depends on FOX genes functions followed by $\mathrm{OPA}_{1}$ enzymes Effects on the produced mitogen from FOX genes activities. The JNK activation occurs through a dual phosphorylation's mechanism of threonine (Thr) and Ser / Tyrosine (Ser/Tyr) residues within Protein-S/T-Thymine-kinase (Thymine kinases) and on mTORC1 (Protein-S/T-Cytosine-kinases which considered $\mathrm{P}_{13} \mathrm{k}$ Akt) subdomain (Figure 2).

The JNK module considered as the basis for adopt, promote, and running proliferations through the mitochondrial $\mathrm{OPA}_{1}$ anabolic oxidative processes which run in highly arranged specific regulations by its enzymes for producing the three types of fattyacyl-CoA gamma, beta, and alpha active subunits for running antiinflammatory pathways, for proliferations processes started by $\mathrm{TLR}_{4}$ synthesis, for re-activating T-cells, and for introducing proper anti-inflammatory processes through modifying and increasing specified cytokine productions, that JNK module steps are basically depending basically on the FOX gene for producing the four kind of protein kinases that depend firstly on the phosphorylation of Ser / Thr mechanism for stimulating and running the necessary mitochondrial anabolic oxidative processes started by gammaoxidation, followed by beta-oxidation and ended by alphaoxidations where the last is specified for proliferations started by $\mathrm{TLR}_{4}$ synthesis which depend on phospholipase effects on linear beta-kinases (beta-subunits) which through feedback and transcription will reactivate linear extracellular regulated kinases ERK1/2. 


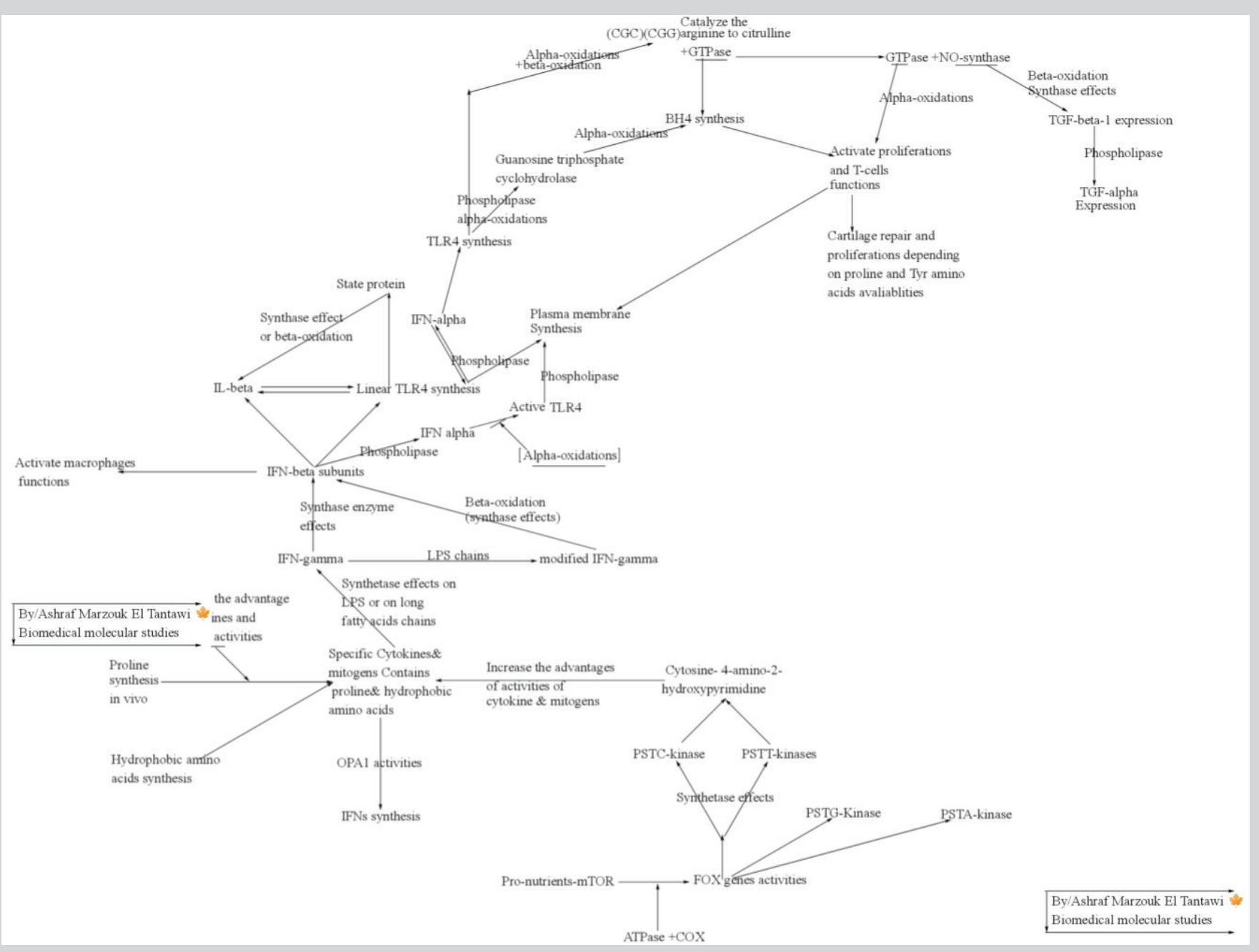

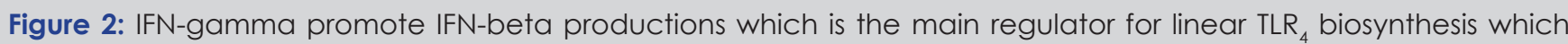
depends on phospholipase for phospholipid and plasma-membrane synthesis.

The JNK is necessary in the regulation of platelet biosynthesis, interleukin-1 productions, interleukin-12 productions, interferongamma, beta, and specifically interferon-alpha (IFN-alpha) productions (Figure 3). The functions and activities of FOX genes are basically specifying for producing the four kind mitogens of kinases that those mitogen kinases will follow the JNK function pass way under the effects of anabolic mitochondrial OPA enzymes $_{1}$ regulations with the availabilities of LPS or long fatty acids molecules chains for producing $\mathrm{TLR}_{4}$ genes, cytokines, for IFNs back productions and for cells proliferation steps. JNK-interacting protein 3 associates with Toll-like receptor 4 and is involved in LPSmediated JNK activation [1].

Where, the protein kinases that produced from FOX activities pathways will follow the effects of mitochondrial anabolic enzymes through JNK with LPS availabilities for $\mathrm{TLR}_{4}$ synthesis upon first running gamma-oxidation by synthetase effects on LPS, then followed by beta-oxidation on gamma-subunits through the effects of synthase for producing linear extracellular signal-regulated beta-kinases $\left(\mathrm{ERK}_{1}\right.$ and $\left.\mathrm{ERK}_{2}\right)$ which upon phospholipase will reactivating the $\mathrm{TLR}_{4}$ synthesis and activities, T-helper 1 type immune response, and Platelet biosynthesis pathways through interferon alpha productions. Phorbol ester is long fatty-acyl-CoA-synthetase active subunits are produced due to effects of synthetase enzyme on long fatty acids chains, which will follow the effect of synthase for producing the long-fatty-acyl-CoA-synthase active subunits which considered as the basis of extracellular signals-regulated betasubunits which necessary for activating the $\mathrm{TLR}_{4}$ synthesis, and for increasing of interferon-beta (Interferon-1) productions [2].

\section{Mitogen-Activated Protein Kinases (MAPK) are Basically Produced by Atpase and COX Followed by FOX Functions}

MAPK stored or located on human chromosomes but originally formed through FOX genes activities regulated by ATPase and OPA activities, where MAPK encodes kinases proteins of amino acids regulated by mitochondrial $\mathrm{OPA}_{1}$ enzymes and by ATPase activities. MAPK is proteins kinases that characterized by the presence of specified amino acids (in specific qualities and quantities in specific arrangements) In addition to the importance of presence of the serine / threonine is for running specific phosphorylation's mechanism on those two amino acids for Brocken and separating their triplets' nucleotides $\{\{$ TCT - Ser

$$
\begin{aligned}
& \text { TCC - Ser } \\
& \text { TCA - Ser } \\
& \text { TCG - Ser } \\
& \text { ACC - Thr }
\end{aligned}
$$


ACA - Thr

ACG - Thr\}\} for producing the four types of basic kinases:

1. protein-Ser /Thr -Thymine kinases (PSTT-K)
2. protein-Ser /Thr -guanine kinases (PSTG-k)

3. protein-Ser /Thr -Cytosine kinases (PSTC-k or mTORC ) $^{2}$

4. protein-Ser /Thr -adenosine kinases (PSTA-k),

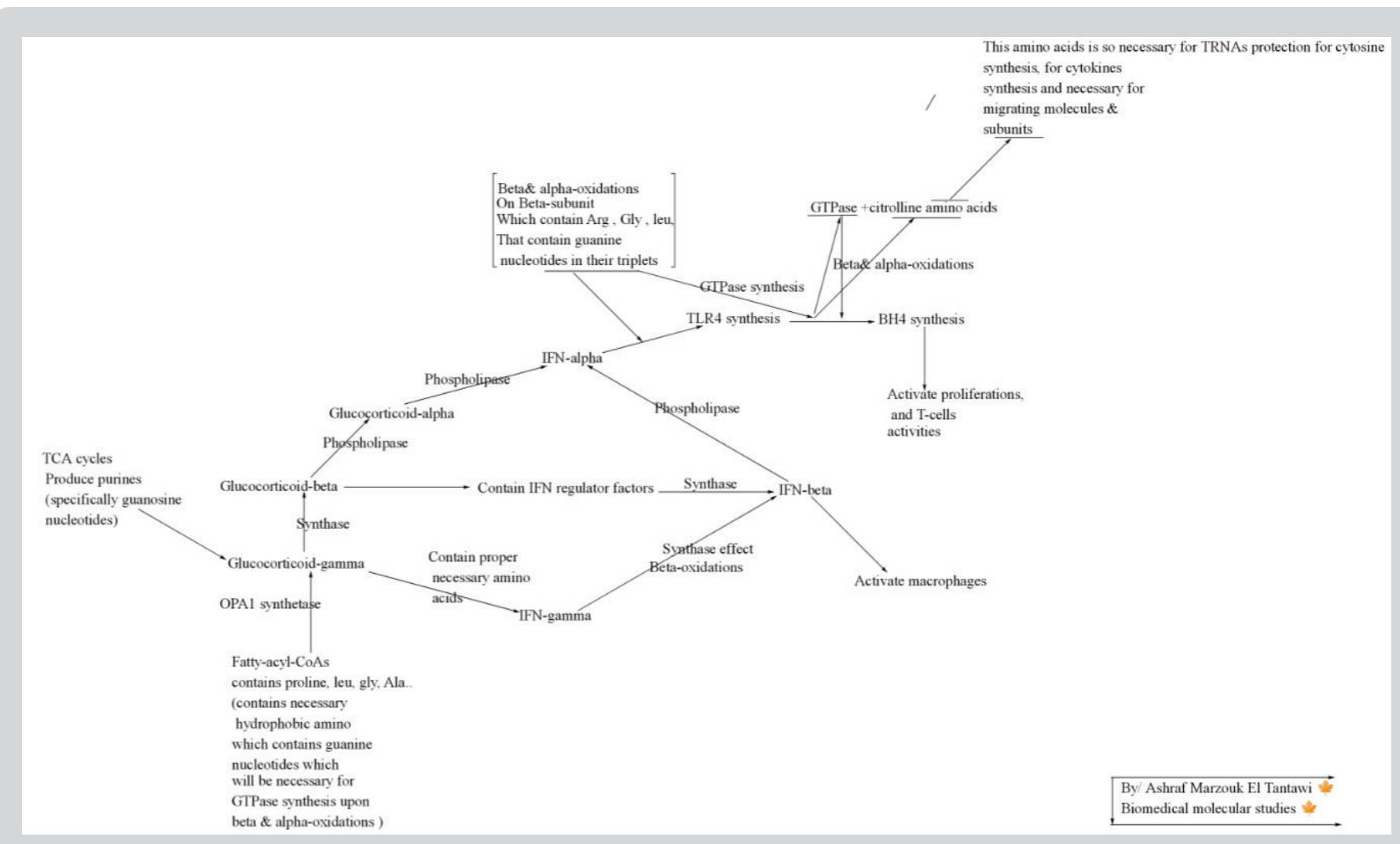

Figure 3: Both Glucocorticoids isoforms subunits and IFNs (IFN-gamma, IFN-beta \& IFN-alpha) are having the responsibilities of recovering each other's \& GTPase production for $\mathrm{BH}_{4}$ synthesis during cellular cycles in vivo.

Notice the main necessary nucleotide in Ser is the Thymine nucleotides where deficiency of Thymine will lead deficiency in PSTT-K and PSTC-k productions and will reflect decreasing in estrogen synthesis with increasing in androgen (where the main nucleotide in androgen is adenosine with almost absence of thymine nucleotides), that will reflect diabetic health problems.

MAPK are mainly and formed by FOX activities in main four kinds of kinases produced during the activities and functions of FOX genes that will follow the mitochondrial anabolic enzymes effects (synthetase, synthase, and phospholipase) for modifying the produced four kind of protein kinases PSTGk, PSTCk, PSTTK, and PSTA Kinase, where their functions and their advantages of specificities depends on their amino acids composition and their arrangement in specific sequences, which will appear in vivo as specific mitogen that necessary for reactivating RORs isoforms synthesis and for producing the three Fatty Acyl-Coenzyme-A isoforms (gamma, beta, and alpha) for running specified cycles for anti-inflammation processes and for cellular proliferation processes.

The MAK productions by FOX genes activities can follow the JNK signaling cascade upon the effects of mitochondrial anabolic enzymes for activating mitochondrial anabolic oxidative processes for the TLR $\mathrm{TL}_{4}$ biosynthesis in the availabilities of Lipopolysaccharide (LPS) and long fatty acids chains (LFC), for $\mathrm{TLR}_{4}$ synthesis for S6K protein synthesis and for NF- $\mathrm{kB}$ production which depending on the three necessary active-lipo-proteins subunits (isoforms) productions upon $\mathrm{OPA}_{1}$ activities:

1. fatty-acyl-CoA-synthetase (gamma-subunits)

2. fatty-acyl-CoA-synthase (beta-subunits)

3 Fatty-acyl-CoA-phospholipase (alpha-subunits).

The advantage of origin, quality, quantity, of each amino acids are characterizing the mitogen kinases subunits functions and activities, and hormones composition activities, Where, the availabilities of proline $\left(\mathrm{C}_{5} \mathrm{H}_{9} \mathrm{NO}\right)$ which can be produced from TCA cycles in vivo "and from the conversion of glutaric acid to ketoglutarate then to glutamate" can activate Hippo pathways and $\mathrm{TLR}_{4}$ synthesis by promoting the $\mathrm{OPA}_{1}$ anabolic oxidative processes (gamma-oxidation and beta-oxidations upon synthetase then synthase respectively ) which needed for $\mathrm{TLR}_{4}$ productions , for the transforming growth factor-beta (TGF- $\beta$ ) productions, and for interleukin-beta production, where TGF- $\beta_{1}$ productions started firstly by TGF-gamma productions upon first the effects of synthetase enzyme on long fatty acids chains, on LPS, and on fatty phospho-lipo-proteins (which produced from ATPase and COX oxidative activities for producing prostaglandins ), then will be following the effect of synthase enzyme on fatty acyl-CoA-synthetase for producing proper fatty-acyl-CoA-synthase, which considered as beta-subunits (with specific composition of amino acids) that produced by beta-oxidation (synthase effects ) that necessary 
for Interleukin-beta production and for TGF- $\beta$ productions for adopting cellular and extracellular-signals regulated kinase (ERK1/2)activities, for adopting anti-inflammatory processes and then for running the cells proliferations upon the phospholipase effects on IL-beta and on Beta-subunits.

\section{Proline, ARG and Hydrophobic Amino Acids EG RYR and LEU are so imp to be Involved in IL-Beta and in TLR $_{4}$ Composition}

It's true that Proline does not contain the amino group - NH, but is containing active -ve oxygen linkages that can stimulate and promote ATPase and stimulate gamma-oxidation easily followed by beta-oxidations, then alpha-oxidation, and at the main time able to reactivate the idle inactive +ve linkages (sub-fragments) in kinases proteins molecules for specific modified mitogen protein kinases productions through FOX genes functions, then followed by OPA activities, where the effects of $\mathrm{OPA}_{1}$-synthetase enzyme is carry the function of hydrophobic acids synthesis by the effects of synthetase enzyme through running Gamma-oxidations for creating specific active gamma-subunits, followed by the effects of synthase for producing active beta-subunits through running beta-oxidations for producing Beta-subunit for the further synthesis of alphasubunits through alpha-oxidations effects by phospholipase effects on Beta-subunits where alpha oxidations are so necessary for cells proliferation, for phospholipid bio-synthesis, for promoting T-cells synthesis, and for cells proliferations processes.

The synthesis of proline in vivo is so imp for adopting purines synthesised from TCA cycles, where proline is related to and depending on the synthetase activities for pyrimidine synthesis, and in the main time stimulate synthetase activities and Thymine synthesis is for adopting the production of purines (from TCA cycles) upon the synthetase effects on long fatty acid chains, and also proline biosynthesis in vivo is so necessary for reactivating macrophages and T-cells where due to the conversion of glutamate to ketoglutarate then to glutamate results of the consuming $\mathrm{NH}$ for creating and producing NO-S upon synthase effects (beta-oxidation) which is imp for reactivating macrophages, and also necessary for hydroxy-proline productions. The hydroxyproline is an accurate index of collagen content and production as well as normal lung tissue [3]. Also, Proline provides in vivo site-specific flexibility for collagen [4].

Proline plays an necessary important roles in $\mathrm{TLR}_{4}$ and TGFbeta/alpha synthesis and activities, that the presence of proline in $\mathrm{TLR}_{4}$ and in TGF-beta will possess dpecofic advantages in cellular processes and will direct the flow of biological reactions to collagen and blood platelets synthesis, where proline plays imp functions in plant growth and in collagen flexibility, that availabilities of proline in $\mathrm{TLR}_{4}$ genes and in TGF-beta will accelerate gamma-oxidation, then beta, then alpha-oxidations for alpha-subunits production that will give flexibility to tissues, blood platelet and collagen synthesis.

The Proline biosynthesis in vivo is a determinant of imp keys of many cells' membranes and proteins activities, where also proline plays important roles in increasing signals activities, in increasing anabolic oxidative processes by OPA enzymes, and in increasing plants developments through their cells walls developments. The proline biosynthesis in vivo is depending on TCA cycles functions and on the availabilities of glutatarate and succinyl-CoA, where the role of the production of hydroxyproline and the proline-rich proteins are important for the characterizing cell-membranes proteins structures and proline play pivotal roles in cell signal transduction activities throughout characterizing the ERK1/2 activities and $\mathrm{TLR}_{4}$ biosynthesis.

Decreasing in Ser amino acids and deficiency in proline synthesis in vivo lead to decreasing in pyrimidine synthesis, that will not adopt the activities of TCA cycles which produce purines and, in many cases, can lead to decreasing in vitro D that will reflect less absorption in intestine. Proline imp for producing soluble Cytosine nucleotides Linked actively to each of other for cytokine production (CCC, CCG, CCA. CCT) which have mainly advantaged to migrations characteristics and increase cytokine activities. Availability of two Cytosines in cytokines are so imp for the migrations characters of molecules and for cytokine synthesis result of the formation of cytosine- 4-amino-2-hydroxypyrimidine.

Cytokines including the chemokines, interferons (INFs), interleukins, lymphokines, and tumor necrosis factors (TNF), are Basically, contains hydrophobic amino acids chains for contains the kinases produced by FOX genes pathways activities where some of those kinases produced from FOX actually can be linked together for producing specific kind of cytokine contain advantages for proper cellular activities, which will follow specific pathways for feeding cells and immune through the Mitochondrial OPA 1 gene proper activities, where those kinases or mitogen can produced back by a range of cells activities including immune cells like macrophages, $\mathrm{B}$ lymphocytes, T lymphocytes and mast cells, as well as endothelial cells, fibroblasts, and various stromal cells.

The advantages of the presence proline in Cytokine's molecules are promoting the maturation, cells growth, and responsiveness of cell populations but through mitochondrial regulations and modification for producing gamma-subunits then beta-subunits upon effects of synthetase and synthase respectively. I consider that the tissues that produce cytokines are the tissues of the FOX genes activities for producing the four kinases' proteins, where cytosine, guanine, and thymine proteins kinases are the basis of imp necessary active protein kinases for cytokines biosynthesis, for IFNs biosynthesis, for antigen synthesis and for IL-beta productions.

The deficiency in proline biosynthesis and in pro-nutrientsmTOR Will reflect decreasing in cytokine proper activities, (where proline can promote the formation of cytosine- 4-amino2-hydroxypyrimidine which can increase the molecules migration and their flexibilities and their proper activities) Lead to decreasing in the anabolic oxidations and increase the pathogenic mutations in IFNs molecules, lead to the Revival the viral emissions in vivo. Cytokines can be modified "improved "in the Golgi (but not synthesis in Golgi) that as I mentioned before that cytokines are basically depending on FOX functions followed by OPA enzymes activities, and their advantages depending on the quality and quantity of specific amino acids in their active subunits, where cytokines can traffic through the endoplasmic reticulum as soluble mediators (specifically if contain proline), and they remain to modulate membranes binding and membrane functions, and they can be produced into cytosolic active forms that can be migrated for further modification by mitochondrial $\mathrm{OPA}_{1}$ activities, that can be returning to nucleus that can act as transcriptional regulators for promoting mitochondrial oxidative processes : firstly Gammaoxidations then by beta-oxidation respectively by $\mathrm{OPA}_{1}$ enzymes functions.

Where, Cytokines bind to reciprocal receptors which prepared originally by $\mathrm{OPA}_{1}$ enzymes for producing the fatty-acyl-CoAsubunits (isoforms) receptors that reside either on the membrane 
of a target cell or in the soluble phase Membrane receptors, and can drive novel gene expression to promote effector functions plus can increase host defenses by up-regulating and improvement the antigen presentation, but through either the protein amino acids subunits or through the structure of fatty-acyl-CoA gamma or beta then alpha-active linear subunits. The cytosine-4-amino-2hydroxypyrimidine $\left(\mathrm{C}_{4} \mathrm{~A}_{2} \mathrm{HP}\right)$ is basically its synthesis depend on two kinases protein which are cytosine-Ser /Thr kinases (ProteinS/T-Cytosine-kinase ) and protein-Ser/Thr-Thymine-kinase (PSTTKinase), where the $\left(\mathrm{C}_{4} \mathrm{~A}_{2} \mathrm{HP}\right)$ considered as the main basics of IFNs compositions and main for protein $\mathrm{C}$ components, where deficiency in $\left(\mathrm{C}_{4} \mathrm{~A}_{2} \mathrm{HP}\right)$ will lead to deficiency in IFNs activities and in protein $\mathrm{C}$ biosynthesis lead to decreasing or deficiency in the alveolar epithelium as a dynamic barrier in pathogenesis.

\section{IFN-Gamma Promote IFN-Beta Production upon Synthase Effects which is the Main Regulator for Linear TLR $_{4}$ Biosynthesis which Depend on Phospholipase for Phospholipid Membranes Synthesis}

The biosynthesis of IFNs started by IFN-gamma which depend on synthetase effects followed by IFN-beta (which depend on synthase) then IFN-alpha (which depend on phospholipase) respectively, where last beta and alpha-oxidations are the mirror of re-activating TLRs genes synthesis, where IFN-alpha enhanced TLR responsiveness in macrophages by up-regulating the expression of $\mathrm{TLR}_{3}, \mathrm{TLR}_{4}$, and $\mathrm{TLR}_{7}[5]$.

Interferon-alpha (IFN alpha) and interferon-gamma (IFN gamma) each induced in susceptible target cells a state of resistance to viral replication and can reduced cellular proliferation, presumably through different mechanisms [6]. Interferons are basically cytokines depending on FOX genes and followed by effect of mitochondrial $\mathrm{OPA}_{1}$ enzymes functions, where after cytokine production upon FOX activities will be modified by the effects of mitochondrial enzymes to produce specific modified IFN-gamma with specific amino acids hydrophobic acids in specific sequences upon synthetase enzymes, then will be followed the synthase effects on IFN-gamma for prod icing modified IFN-beta (upon the effects of synthase) which will confer its resistance in functions to viral infections and considered to be imp for linear $\mathrm{TLR}_{4}$ synthesis, then will follow alpha oxidations for producing IFNalpha (upon phospholipase effects) which has the responsibility for $\mathrm{TLR}_{4}$ biosynthesis, where each of IFN structure has own steps in functions and activities, and all IFN subunits isoforms structures are depending on each other's for strengthen and running immune activities for anti-inflammation activities and for potentiate macrophages and T-cells activities were, Interferons are related cytokines identified by their ability to confer resistance to viral infections [7].

The Defective IFN- $\beta$ signaling (may be due to decreasing in proline, in Arg or in necessary hydrophobic amino acids) are having the responsibility for Lower Ability of BALB/c Macrophages to Produce NO in Response to LPS [8]. The availabilities of proline and other necessary active hydrophobic amino acids in IFN-beta subunits molecules are so imp and responsible for determining the advantages of IFN- $\beta$ activities and TLR Th $_{4}$ biosynthesis too (which produced upon phospholipase effects).

But at the main time the values of amino acids in quality and in quantity containment in each IFN isoform subunits can determine its own advantages in activities of its own subunits functions depending on its composition from proline, Arg, and from necessary hydrophobic amino acids, that each IFN subunit can re-start its own basic activity for reproducing its own active subunits chain , then will start to activate the other two IFNs subunits again, e.g.: in IFNs biosynthesis when IFN- $\beta$ increases will increase its own betaoxidations that will increase the Antiviral effect then will potentiate the macrophages activities (but in availability of proline, Arg and necessary hydrophobic acids), then will activate and potentiate the $\mathrm{TLR}_{4}$ synthesis and IFN-alpha activities in orders, while the reincreasing in IFN-gamma will increase its own gamma-oxidation for its own modified gamma-subunits reproduction that can cause a temporary suppression to other two IFNs producers and to TLR synthesis and will reactivate macrophages activities [9], due to the IFN-gamma activities will increase its own gamma-oxidation for analyzing membranes and inflammations for reactivating firstly pyrimidine synthesis for hydrophobic acids synthesis for reproducing its own new modified IFN-gamma subunits (in availability of LPS) and for adopting purines and pyrimidines in vivo, that upon synthase enzyme (beta-oxidation) will re-activate IFN-beta subunits productions which can potentiate macrophage activity and anti-inflammation processes, then upon phospholipase effects on IFN-beta will activate alpha oxidations for IFN-alpha productions which will reactivate $\mathrm{TLR}_{4}$ synthesis (also depend on phospholipase effects), which has the anti-inflammatory function too and can reproducing IFN-beta again throughout the feedback upon $\mathrm{OPA}_{1}$-synthase enzyme effects (through feedback of betaoxidation).

The protein $C$ system provides important functions and control of blood coagulation where proteins $\mathrm{C}$ basically are the necessary regulator for fatty-acyl-CoA-synthetase synthesis and for IFNgamma-subunits productions upon synthetase effects. That both Vitamin E \& K-dependent protein C are the key components of anticoagulant serine protease. And therefore, vit $\mathrm{E}$ and $\mathrm{k}$ are modulated first in vivo their own necessary proper gamma-subunits synthesis (fatty-acyl-CoA-synthetase subunits) which is the basics for producing IL-beta upon the synthase effects which will regulate $\mathrm{TLR}_{4}$ synthesis upon the phospholipase effects then will produce upon beta and alpha oxidations for reactivating phospholipid membranes synthesis and at the same time act for anticoagulation's [10]. The presence of vitamin $\mathrm{K}$ in the IFNs production for $\mathrm{TLR}_{4}$ gives its advantages for anti- coagulation characters and possess its own cells the ability (which contain vitamin $\mathrm{K}$ and vit $\mathrm{E}$ in their plasma membrane and contains proline and Arg amino acids in their composition) to resist and prevent blood clotting and dissolve it in vivo. Protein C (APC) has a protective effect on mechanical tension and barrier integrity in human alveolar epithelial cells $\left(A_{549}\right)$ exposed to thrombin [11].

The respiratory epithelium is a primary target of an inflammatory/infectious condition at the epithelial-blood interface and is itself capable of amplifying an inflammatory signal for stimulating IFN-gamma and IFN-beta productions, where the IFN-beta will firstly potentiate macrophages activities and antiinflammatory processes the reactivate IFN-alpha production which is the first necessary for cells proliferations.

That inflammatory signals can stimulate IFN-gamma activities then $\mathrm{OPA}_{1}$ functions for restart the gamma, beta, then alphaoxidative processes upon effects of synthetase, then synthase then phospholipase respectively for re-producing IFN-gamma, IFN-beta and for IFN-alpha subunits productions respectively, then for $\mathrm{TLR}_{4}$ synthesis respectively and for $\mathrm{BH}_{4}$ synthesis for recover broken cells membranes and for proliferation. 
As I mentioned before that IFN-gamma alone can suppress both macrophages and $\mathrm{TLR}_{4}$ synthesis and activities even can suppress their production from interleukin (IL-beta), that as IFNgamma Started to be activated as IFN-gamma oxidations will start for analyzing inflammations and available content of molecules for creating their own modified gamma-subunits which contains advantages of specific amino acids in arrangements for recreating step of beta-oxidation for producing the (Beta-subunit) IFN-beta subunits upon the synthase effect which will be responsible for recreating the linear $\mathrm{TLR}_{4}$ which will be responsible for $\mathrm{BH}_{4}$ synthesis and then for Plasma membrane synthesis upon phospholipase effects. Where, in sharp contrast, IFN- $\gamma$ was shown to synergize with TLR agonists for induction of macrophage tumoricidal activity and production of both of both $\mathrm{NO}$ and pro-inflammatory cytokines (TNF- $\alpha$, IL-...), furthermore, IFN- $\gamma$ was shown to suppress macrophage IL-10 secretion induced by TLR agonists [12].

Phospholipase is the Basic Regulated Tool for $\mathrm{TLR}_{4}$ Synthesis Which Act on IFN-Beta Subunits, for Producing TLR $_{4}$, where both are Sharing Together the Responsibility for Producing Growth Factor $\mathrm{BH}_{4}$ for Proliferations

As soon as $\mathrm{TLR}_{4}$ synthesized and the effect of phospholipase continued as the alpha-oxidations process will begin and continued for the Releasing of Guanosine triphosphate Cyclopyrrolone (GCHase) molecules which supposed to contain specific hydrophobic amino acids including proline, were Guanosine triphosphate Cyclopyrrolone considered as the rate-limiting enzyme for tetrahydrobiopterin $\left(\mathrm{BH}_{4}\right)$ synthesis and reactivities, which considered as the growth factor tools for cells proliferation and for T-cells activations. The metabolite $\mathrm{BH}_{4}$ controls $\mathrm{T}$ cell proliferation in autoimmunity [13].

In other way and other clear meaning that the synthesis of $\mathrm{TLR}_{4}$ in proper active structures with availability of proper phospholipase are the basic tools for growth factor $\mathrm{BH}_{4}$ productivity, where its synthesis is depending on to beta-oxidation followed by alpha-oxidations phosphorylation processes on Arg amino acids for taking guanine nucleotides for releasing Guanosine triphosphate Cyclohydrolase (GTP-Chase) and release citrulline where the GTPChase is necessary for $\mathrm{BH}_{4}$ modulations.

At deficiency in GTP-Chase synthesis will lead to dysfunction in $\mathrm{BH}_{4}$ production, where Patients with recessive GTP-CH deficiency demonstrate much more neurologic dysfunction than the dominant form, with early-onset developmental delay, seizures, tremors, truncal hypotonia with appendicular hypertonia, and autonomic dysfunction [14].

I would like to add that deficiency in amino acids which contains Guanine nucleotides in their triplets as Arg, cys, leu, Ala, Val. will lead to deficiency in the GTP-Chase productions and may can strongly be related to deficiency in TCA cycles which necessary for purines production. Both IFN-beta and glucocorticoid-beta are designed anti-inflammatory subunits are depending on the activities of synthase enzyme within $\mathrm{OPA}_{1}$ mitochondrial membrane for producing the long fatty acyl-CoA-synthase (Betasubunit) with specific compositions and sequences from amino acids that can determine their advantages in immunity functions. Both glucocorticoid-beta and IFN-beta can recover each other's in their different tissues, that Glucocorticoids Conditioning of Myeloid Progenitors can Enhance TLR 4 Signaling [15]. Stimulated Glucocorticoid-beta Subunits in a proper biological environment in vivo can stimulate and recover $\mathrm{TLR}_{4}$ synthesis and then the $\mathrm{BH}_{4}$ proliferations factor in the availabilities of proper active sequences of $\mathrm{OPA}_{1}$ synthase and phospholipase, where the both pervious enzymes can reactivate the beta-oxidations and alpha-oxidations necessary for producing GTP-Chase and for reactivating $\mathrm{P}_{13} \mathrm{~K}$ which is necessary for $\mathrm{BH}_{4}$ synthesis, where beta and alpha-oxidations can catalyze Arg (and other amino acids which contain guanine) for using their guanine nucleotides for producing GTP-Chase enzymes (and citrulline) for enhancement the $\mathrm{TLR}_{4}$ productions and then for $\mathrm{BH}_{4}$ productions for proliferation processes.

Also, promoter of the human glucocorticoid receptor (hGR) gene contains an interferon (IFN) regulatory factor element (IRF-E) [16], That at recovery of IFN, the RF-IFN will be activated first by transcriptions then through beta-oxidations for reproducing IFNbeta which under effect of alpha-oxidations will recover the TLR synthesis for necessary proliferations, for plasma membrane synthesis and for macrophages and T-cells reactivation.

Erythropoietin is glycoprotein synthesized in the liver, but it looks like its production switches almost exclusively to the kidney, but facts ensue that Erythropoietin is filtered and reextracted through renal tubules, where red blood cells carry west toxic molecules including positive molecules to the kidney, where Erythropoietin need to be re-extracted by renal tubules in kidney due to its importance and values in its compositions to cells proliferations.

As Erythropoietin decreased as $\mathrm{BH}_{4}$ biosynthesis in liver will be Increased for recover the decreased Erythropoietin, that will reflect increasing in $\mathrm{TLR}_{4}$ re-activities and production and increasing in GTP-Chase productions which result from catalyzing the Arg (CGG, CGA) amino acids for releasing GTP-Chase enzymes \& the citrulline (that I consider citrulline is originally cytosine nucleotides which basically bonded to guanine in Arg triplets) which will be used for reactivating the Akt $\left(\mathrm{PI}_{3} \mathrm{k}\right)$ for reproducing Erythropoietin again.

Where, Activation of phosphatidylinositol 3-kinase $\left(\mathrm{PI}_{3} \mathrm{~K}\right)$ is important for erythropoietin-induced erythropoiesis from $\mathrm{CD}_{34}(+)$ hematopoietic progenitor cells [17] Now, one of the important roles for $\mathrm{PI}_{3} \mathrm{~K}$ (which has been modified by synthase and by phospholipase through beta-oxidations and alpha-oxidations respectively) is the reactivating and accelerating $\mathrm{TLR}_{4}$ for re-peoducing $\mathrm{BH}_{4}$ which is necessary for EPO-induced survival, proliferation, and maturation of early erythroid progenitors. Also, the site of $23 \mathrm{~S}$ rRNA ribozyme, 0 , was both necessary and sufficient to trigger $\mathrm{TLR}_{13}$-dependent interleukin-1 $\beta$ production [18].

That $\mathrm{P}_{13} \mathrm{~K}$ are basics of $\mathrm{S}_{6} \mathrm{~K}$ and $23 \mathrm{~S}$ ribosomal genes productions and repairs which are carrying necessary roles in TLR synthesis and $8 \mathrm{n} \mathrm{BH}_{4}$ productions with advantages composition from necessary active amino acids.

\section{CONCLUSION}

Toll-like receptor-4 $\left(\mathrm{TLR}_{4}\right)$, synthesis is regulated by JNK signaling, by three glucocorticoids isoforms, and by the three interferons isoforms, also depending on availabilities of LPS \& on long fatty acids chains with Arg and proline availabilities. Proline can accelerate anabolic oxidative processes by $\mathrm{OPA}_{1}$ enzymes and provides site-specific flexibility for collagen synthesis in vivo and plays a necessary important role in $\mathrm{TLR}_{4}$ and TGF-gamma/beta/\& alpha synthesis and activities. Phosphatidylinositol 3-kinase $\left(\mathrm{PI}_{3} \mathrm{~K}\right)$ is important for erythropoietin-induced erythropoiesis reactivations, and we have considered that $\mathrm{P} 1_{3} \mathrm{~K}$ are basics of $\mathrm{S}_{6} \mathrm{~K}$ and $23 \mathrm{~S}$ ribosomal genes productions and repairs which are 
carrying necessary roles for TLR synthesis and $8 \mathrm{n} \mathrm{BH}_{4}$ productions with advantages of containment of necessary active amino acids e.g.: proline, Arg, leu, tyr...etc.

Both IFN-beta and glucocorticoid-beta are designed antiinflammatory subunits are depending on each other's and on the activities of $\mathrm{OPA}_{1}$-synthase enzyme for producing the long fatty acyl-CoA-synthase (Beta-subunit) with specific compositions and sequences from amino acids which can determine their advantages in immunity functions e.g., their containment of tyr, proline, Arg gly. etc., where, both glucocorticoid-beta and IFN-beta can recover each other's in their different tissues, and in almost general fact the IFNs are related to glucocorticoids subunits and are covering each other in vivo. The containment of Arg, and prolines amino acids in genes and in active fatty-acyl-CoA-subunit isoforms including glucocorticoids and interferons three subunits can accelerate anabolic $\mathrm{OPA}_{1}$ oxidative processes and can save living cells and their tissue from hypoxia.

\section{REFERENCES}

1. Tetsuya M, Akio MK, Yasunobu Y (2003) JNK-interacting protein 3 associates with toll-like receptor 4 and is involved in LPS-mediated JNK activation. EMBO J 22(17): 4455-4464.

2. Momose I, Terashima $M$, Nakashima $Y$, Sakamoto $M$, Ishino $H$, et al. (2000) Phorbol ester synergistically increases interferon regulatory factor-1 and inducible nitric oxide synthase induction in interferongamma-treated RAW 264.7 cells. Biochim Biophys Acta 1498(1): 19-31.

3. Kehrer JP, Lee YC, Solem SM (1986) Comparison of in vitro and in vivo rates of collagen synthesis in normal and damaged lung tissue. Exp Lung Res 10(2): 187-201.

4. Chow WY, Forman CJ, Bihan D, Anna MP, Rakesh R, et al. (2018) Proline provides site-specific flexibility for in vivo collagen. Sci Rep 8(1): 13809.

5. Sirén J, Pirhonen J, Julkunen I, Matikainen S (2005) IFN-alpha regulates TLR-dependent gene expression of IFN-alpha, IFN-beta, IL-28, and IL29. J Immunol 174(4): 1932-1937.

6. Levy DE, Lew DJ, Decker T, Kessler DS, Darnell JE, et al. (1990) Synergistic interaction between interferon-alpha and interferon-gamma through induced synthesis of one subunit of the transcription factor $\mathrm{ISGF}_{3} . \mathrm{EMBO}$ J 9(4): 1105-1111.
7. Graber JJ, Dhib JS (2014) Interferons. Reference module in neuroscience and biobehavioral psychology encyclopedia of the neurological sciences ( $2^{\text {nd }}$ edn) $: 718-723$

8. Oliveira LS, Queiroz de NMGP, Veloso LVS, Moreira TG, Oliveira FS, et al. (2014) A Defective TLR4 signaling for IFN- $\beta$ expression is responsible for the innately lower ability of BALB/c macrophages to produce NO in response to LPS as compared to C57BL/6. PLoS ONE 9(6): e98913.

9. Kang K, Bachu M, Park S, Keunsoo K, Seyeon Bae, et al. (2019) IFN- $\gamma$ selectively suppresses a subset of TLR4-activated genes and enhancers to potentiate macrophage activation. Nat Commun 10: 3320.

10. Booth SL, Golly I, Sacheck JM, Roubenoff R, Dallal GE, et al. (2004) Effect of vitamin E supplementation on vitamin $\mathrm{K}$ status in adults with normal coagulation status. Am J Clin Nutr 80(1): 143-148.

11. Puig F, Fuster G, Adda M, Blanch L, Farre R, et al. (2013) Barrier protective effects of activated protein $C$ in human alveolar epithelial cells. PLOS ONE 8(2): e56965.

12. Elisabeth M, Panagiotis FC, Sanjib H, Anna L, Kahsai B, et al. (2017) Toll like receptor ligands and interferon- $\gamma$ synergize for induction of antitumor M1 macrophages. Font Immunol 26(1).

13. Cronin SJF, Seehus C, Weidinger A, Talbot S, Reissig S, et al. (2018) The metabolite $\mathrm{BH}_{4}$ controls $\mathrm{T}$ cell proliferation in autoimmunity and cancer. Nature 563(7732): 564-568.

14. Raben DM (2013) Phosphofructokinase-2/Fructose Bisphosphatase-2. Amino Acid Metabolism: 456-458.

15. Tian YZ, Raymond AD (2017) Glucocorticoid conditioning of myeloid progenitors enhances $\mathrm{TLR}_{4}$ signaling via negative regulation of the phosphatidylinositol 3-Kinase-Akt pathway. J Immunol 178 (4): 25172526.

16. Nunez BS, Geng CD, Pedersen KB, Millro MCD, Vedeckis WV, et al. (2005) Interaction between the interferon signaling pathway and the human glucocorticoid receptor gene 1A promoter. Endocrinology 146(3): 14491457.

17. Myklebust JH, Blomhoff HK, Rusten LS, Stokke T, Smeland EB, et al. (2002) Activation of phosphatidylinositol 3-kinase is important for erythropoietin-induced erythropoiesis from CD34(+) hematopoietic progenitor cells. Exp Hematol 30(9): 990-1000.

18. Xiao DLi, Zhijian JC (2012) Sequence specific detection of bacterial 23S ribosomal RNA by TLR13. Cell biology, Immunology and Inflammation, Elife 30(1): e00102. 